\title{
Data Set Homeomorphism Transformation Based Meta-clustering*
}

\author{
Xianchao Zhang, Yu Zong, He Jiang, and Xinyue Liu \\ School of Software, Dalian University of Technology, Dalian 116621, China \\ \{xczhang, zongyu, jianghe, xyliu\}@dlut.edu.cn
}

\begin{abstract}
Clustering analysis is an important data mining technique with a variety of applications. In this paper, the data set is treated in a dynamic way and a Data Set Homeomorphism Transformation Based Meta-Clustering algorithm (DSHTBMC) is proposed. DSHTBMC decomposes the task of clustering into multiple stages. It firstly constructs a series of homeomorphous data sets ranging from high regularity to low, and then iteratively clusters each homeomorphism data set based on the clustering result of the preceding homeomorphism data set. Since data sets of high regularities are easier to be clustered, and the clustering result of each homeomorphism data set can be used to induce high quality clusters in the following-up homeomorphism data set, in this way, the hardness of the problem is decreased. Two strategies (i.e., Displacement and Noising) for data set homeomorphism transformation are proposed, with classical hierarchical divisive method--Bisecting k-means as DSHTBMC's subordinate clustering algorithm, two new clustering algorithms---HD-DSHTBMC-D and HD-DSHTBMC-N are obtained. Experimental results indicate that the new clustering algorithms are remarkably better than Bisecting k-means algorithm in terms of clustering quality.
\end{abstract}

Keywords: Clustering Analysis, Meta-Clustering, Data Set Homeomorphism Transformation.

\section{Introduction}

Clustering analysis is an important data mining technique with a variety of applications in massive data statistic, network analysis and medicinal graph automatic detection, etc. Clustering divides the data set into different clusters according to the inherent characteristic of data, making data elements similar in the same cluster and dissimilar among different clusters. The recent study progress is proposed in [1-2]. Since the 1940's, a large number of clustering algorithms have been proposed, such as divisive method $\left(\mathrm{k}\right.$-means ${ }^{[3]}$, CLARANS $^{[4]}$, FREM $\left.^{[5]}\right)$, hierarchical method $\left(\mathrm{CHAMELEON}^{[6]}, \mathrm{BRICH}^{[7]}, \mathrm{PDDP}^{[8,9]}\right.$ ) 、 grid-based method (WaveCluster ${ }^{[10]}$,

\footnotetext{
This paper is supported by National of Science Foundation of China under grant number: 60503003.
} 
STING $^{[11]}$, CLIQUE $^{[12]}$ ) and density-based method (DBSCAN ${ }^{[13]}$, OPTICS ${ }^{[14]}$ ). These algorithms do not change the data set being processed, and they are here called static algorithms.

In this paper, a clustering strategy is proposed, which transforms data elements' positions in the data set and clusters the data set in different stages of the position transformation. Thus a Data Set Homeomorphism Transformation Based MetaClustering (DSHTBMC) is obtained. Different from traditional static clustering algorithms, DSHTBMC firstly adopts a data set homeomorphism transformation strategy to construct a series of homeomorphous data sets ranging from high regularity to low, and then, by iteratively calling a subordinate clustering algorithm, clusters each homeomorphism data set based on the clustering result of the preceding homeomorphism data set. Here regularity means the regular extent of data elements distribution. Since data sets of high regularity are easier to be clustered, and the clustering result of each homeomorphism data set can be viewed as a pre-clustering of the next homeomorphism data set, which induces high quality clusters in the latter one. In the end, a high quality clustering result is obtained in the original data set.

Two strategies for data set homeomorphism transformation are proposed in this paper. Displacement method views the extent of data elements closing to the barycenter of the data set as regularity, and constructs homeomorphism data sets by removing data elements from nearby barycenter to their initial positions gradually. Noising method views the even extent of data element distribution as regularity, and constructs homeomorphism data sets by adding random noises ranging from strong to weak to the original data set, where the noises are used reduce data elements' differences. Different transformation strategies lead to different DSHTBMCs and the DSHTBMCs adopting the above two strategies are called DSHTBMC-D and DSHTBMC-N respectively. The classical hierarchical divisive method--Bisecting kmeans ${ }^{[15]}$ is used as DSHTBMC's subordinate clustering algorithm, and two new clustering algorithms----HD-DSHTBMC-D (Hierarchical Divisive DSHTBMC Using Displacement ) and HD-DSHTBMC-N (Hierarchical Divisive DSHTBMC Using Noises) are obtained. The performance differences among the new algorithms and the traditional algorithm Bisecting k-means are demonstrated with a series of experiments. Experimental results indicate that the new clustering algorithms are remarkably better than Bisecting k-means algorithm in terms of clustering quality.

This paper is organized as follows. In the next section, relevant notations and definitions are introduced. In section 3 the framework of Data Set Homeomorphism Transformation Based Meta-Clustering (DSHTBMC) and two homeomorphism transformation strategies are described. The principle and framework of hierarchical divisive DSHTBMC algorithm is discussed in Section 4. In the last section, experimental results and performance evaluations are demonstrated.

\section{Preliminaries}

Definition 1. For given data set $X=\left\{x_{1}, x_{2}, \cdots, x_{n}\right\}, \forall i \in\{1,2, \cdots, n\}, x_{i}=\left(x_{i l}, x_{i 2}, \ldots, x_{i p}\right)$ is called a data element of $X$, and $\forall j \in\{1,2, \cdots, p\}, x_{i j}$ is an attribute of $x_{i}$. 
Definition 2. For given data set $X$, clustering is a process of partitioning $X$ into subsets $C=\left\{C_{1}, C_{2}, \ldots, C_{k}\right\}$ according to inherent characteristic of data, subject to: $\forall i, j \in\{1,2, \cdots, k\}, C_{i} \neq \phi, C_{j} \neq \phi, C_{i} \cap C_{j}=\phi(i \neq j)$, and $\bigcup_{i=1}^{k} C_{i}=\{1,2, \cdots, n\}$. Where $C_{i}$ is called a cluster, whose size is denoted by $\left|C_{i}\right|$.

Definition 3. For given data set $X=\left\{x_{1}, x_{2}, \cdots ; x_{n}\right\}$, its barycenter is computed by $w^{X}=\left(w_{1}^{X}, w_{2}^{X}, \ldots, w_{p}^{X}\right), \forall j \in\{1,2, \ldots, p\}, w_{j}^{X}=\frac{1}{n} \sum_{i=1}^{n} x_{i j}$.

To assess the performance of clustering algorithms, two modes, external quality evaluation and internal quality evaluation are adopted at present. The former is to compare whether clustering result of an algorithm is consistent with the result obtained by professionals based on domain knowledge. The latter is mainly to compare the qualities of clustering results obtained by different algorithms. Since external quality evaluation is human dependent, most scholars adopt the mode of internal quality evaluation, so does this paper.

Sergio M.Savaresi etc. ${ }^{[16]}$ proposed an internal quality evaluation, considering both similarity inside clusters and dissimilarity among clusters in accordance with the basic principles of clustering. The relevant definitions are as follows.

Assume that the clustering result is depicted as $C=\left\{C_{1}, C_{2}, \ldots, C_{k}\right\}$, and the barycenter of $C_{i}$ is denoted by $w^{C_{i}}$. Similarity inside a cluster is measured by average discrete degree. For a cluster $C_{i}$, its average discrete degree is computed as $e_{i}=1 /\left|C_{i}\right| \sum_{j \in C_{i}}\left\|x_{j}-w^{C_{i}}\right\|^{2}$. Dissimilarity among clusters is measured by distance between clusters, i.e., the minimum value of distances between the barycenters of a cluster $C_{i}$ and the other clusters, denoted by $\hat{d}_{i}=\min _{j}\left(\left\|w^{C_{i}}-w^{C_{j}}\right\|\right)$.

With the above defined average discrete degree and distance, the internal quality can be computed by:

$$
Q\left(C_{1}, C_{2}, \ldots, C_{k}\right)=\sum_{i=1}^{k}\left|C_{i}\right| e_{i} / \hat{d}_{i} n .
$$

The smaller the value $Q\left(C_{1}, C_{2}, \ldots, C_{k}\right)$ is, the better quality of the obtained clustering result is ${ }^{[16]}$.

Definition 4. ${ }^{[17]}$ Assume $f: X \rightarrow Y$ is an one-one mapping, then $f$ is called a homeomorphism transformation from $X$ to $Y$. If such homeomorphism transformation $f$ exists, then $X$ and $Y$ are called homeomorphism equivalence, denoted by $X \cong Y$. Data set $X$ is called original data set, and $Y$ is called a homeomorphism data set of $X$.

Homeomorphism is an important concept in Topology with a variety of applications in mathematics, biology and physics and so on. 


\section{Data Set Homeomorphism Transformation Based Meta-clustering}

\subsection{The Algorithm Framework}

The underlying idea of data set homeomorphism transformation based meta-clustering is: constructing a series of homeomorphous data sets by transforming data positions, and clustering these homeomorphous data sets. The clustering of each homeomorphous uses the clustering result of the preceding data set as an initial solution. To The control the convergence of the algorithm, a homeomorphism factor is used, which reflects the regular degree of the data sets and can be used to. The algorithm terminates when homeomorphism factor reaches small enough.

The framework of DSHTBMC is described in algorithm 1.

\section{Algorithm 1. DSHTBMC}

Input: the original data set $X$, the data set homeomorphism transformation $f$, the initial value of homeomorphism factor $\alpha_{0}$ and its threshold $\tau$, step length $\lambda$.

Output: clustering result.

Begin

(1) $\alpha=\alpha_{0}$;

(2) perform homeomorphism transformation from $X$ by calling $f$, and a homeomorphism data set $Y$ is acquired;

(3) $Y$ is clustered by calling the subroutine clustering algorithm , and clustering result $C$ is obtained;

(4) while $(\alpha>\tau)$

(4.1) $\alpha^{\prime}=\alpha-\lambda ; C^{\prime}=\varnothing$;

(4.2) the homeomrphism data set $Y^{\prime}$ of $X$ is produced by calling $f$;

(4.3) $Y^{\prime}$ is clustered by calling the subroutine clustering algorithm based on the initial solution $C$, and the clustering result $C$ is acquired;

(4.4) $\alpha=\alpha^{\prime}, C=C^{\prime}$;

(5) return $C$;

End.

\subsection{Data Set Homeomorphism Transformation Strategy}

\subsubsection{Displacement}

The principle of displacement is that data elements of original data set $X$ are diffused gradually from nearby barycenter to their original positions, thus a series of homeomorphism data set with different regularities are constructed. Algorithm 2 describes the framework of displacement. It can be observed from algorithm 2 that internal data difference of the obtained homeomorphism data set is largened gradually as the homeomorphism factor varies from big to small. When the homeomorphism factor equals 1 , that is, $y_{i j}=x_{i j}$, the original data set is resumed. 


\section{Algorithm 2. Displacement}

Input: homeomorphism factor $\alpha \geq 1$, original data set $X$ and its barycenter $w^{X}$. Output: homeomorphism data set.

Begin

(1) for each $x_{i} \in X$ do

$$
\begin{aligned}
& \text { for each } x_{i j} \text { do } \\
& \text { if } x_{i j} \geq w_{j}^{X} \text { then } \\
& y_{i j}=w_{j}^{X}+\left(x_{i j}-w_{j}^{X}\right)^{\alpha} ; \\
& \text { else } \\
& y_{i j}=w_{j}^{X}-\left(w_{j}^{X}-x_{i j}\right)^{\alpha} ;
\end{aligned}
$$

(2) return $Y$;

End.

Power function transformation is adopted to transform position of data element $x_{i j}$ in original data set $X$, so data of original data set should be normalized before data set homeomorphism transformation.

\subsubsection{Noising}

The essential idea of noising is to construct a series of homeomorphism data set with different regularities by adding noise from strong to weak to the original data set $X$. Algorithm 3 elaborates the framework of noising. During the course of the homeomorphism factor varying from big to small, the noise decreases gradually, and internal data elements differences of homeomorphism data set get largened little by little. When the homeomorphism factor equals 0 , noise disappears, and then the original data set is resumed.

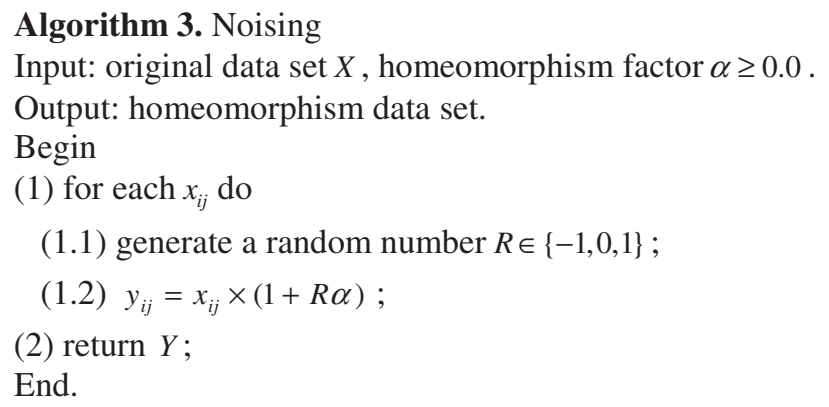

\section{Hierarchical Divisive DSHTBMC}

In this section, the classical hierarchical divisive method--Bisecting k-means is used as subordinate clustering algorithm of DSHTBMC, and a concrete algorithm is proposed.

\subsection{Principle and Framework of the Algorithm}

As an implementation DSHTBMC, we use the idea of hierarchical divisive clustering algorithm, and call this implementation as Hierarchical Divisive DSHTBMC (HDDSHTBMC). 
Hierarchical divisive clustering is a procedure of constructing hierarchical binary tree, where non-leaf nodes of binary tree denote mesne divisive clusters, and leaf nodes denote the clustering result. Correspondingly, HD-DSHTBMC performs clustering on a homeomorphism data sets clustering tree, which is a special hierarchical binary tree with the following features:

(1) The root of the tree corresponds to the initial homeomorphism data set with the highest regularity;

(2) The bottom layer corresponds to original data set, and each leaf node is a cluster;

(3) The rest layers correspond to the other homeomorphism data sets, and in each layer, a node is a cluster of the corresponding data set.

Note that each layer is more regular than the lower layers.

Algorithm 4 gives the framework of HD-DSHTBMC.

\section{Algorithm 4. HD-DSHTBMC}

Input: original data set $X$, the homeomorphism transformation $f$, the initial value of homeomorphism factor $\alpha_{0}$ and its threshold value $\tau$, step length $\lambda$.

Output: clustering result.

Begin

(1) $\alpha=\alpha_{0}$;

(2) call $f$ to produce homeomorphism data set $Y$ of original data set $X$;

(3) the initial cluster $\operatorname{set} C=Y$;

(4) while $(\alpha>\tau)$ do

(4.1) $\alpha^{\prime}=\alpha-\lambda ; C^{\prime}=\varnothing$;

(4.2) call $f$ to produce new homeomorphism data set $Y^{\prime}$;

(4.3) for each $C_{i} \in C$ do

call Bisecting k-means algorithm to perform division operation for $C_{i}$, and insert the result into $C^{\prime}$;

(4.4) $\alpha=\alpha^{\prime} ; C=C^{\prime}$;

(5) return $C$;

End.

Different clustering algorithms are obtained by performing diverse strategies of data set homeomorphism transformation in the layers of the tree. The algorithms adopting displacement and noising are denoted by HD-DSHTBMC-D and HDDSHTBMC-N, respectively.

\section{Experimental Results}

The contrasts of clustering quality, among Bisecting k-means, HD-DSHTBMC-D and HD-DSHTBMC-N, are shown in this section. The parameters used in HDDSHTBMC-D algorithm are: $\alpha_{0}=1.0, \lambda=1, \tau=1$, and in HD-DSHTBMC-N: $\alpha_{0}=1.0, \lambda=0.1, \tau=0.0$.

Fig. 1 compares the qualities of the three algorithms for the same data set of different cluster numbers. The size of the data set is 15000. The three clustering 
algorithms are run and the clustering results, with the number of clusters $4,6,8,10,12,14,16,18$ and 20 are obtained. It can be observed from the figure that the clustering qualities of HD-DSHTBMC-D and HD-DSHTBMC-N are obviously better than Bisecting k-means method. The clustering qualities of HD-DSHTBMC-D and HD-DSHTBMC-N are very close, while HD-DSHTBMC-N is slightly better.

Fig. 2 compares the qualities of the three algorithms under the same cluster number on data sets of different sizes. The sizes of data sets are 1000, 3000, 5000, 7000, $9000,11000,13000,15000$. The three different clustering algorithms divide the data sets into 8 clusters. It can be concluded from the figure that the clustering qualities of HD-DSHTBMC-D and HD-DSHTBMC-N are remarkably better than Bisecting kmeans method without reference to data size.

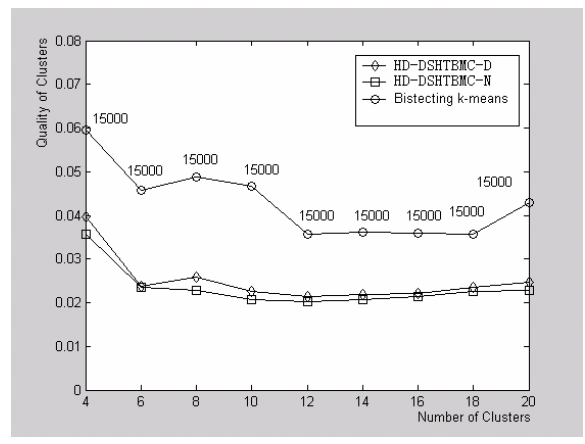

Fig. 1. Comparison of Cluster Quality of 3 Algorithms on the Same Data Set with Different Cluster Numbers

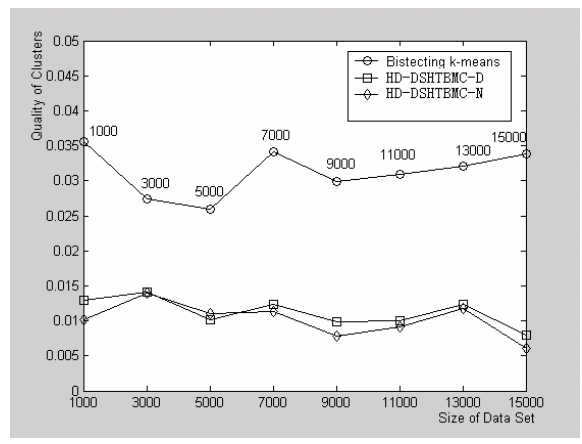

Fig. 2. Comparison of Cluster Quality of 3 Algorithms under the Same Cluster Number on Different Data Sets

\section{Conclusion}

Rather than concrete algorithms, the main contribution of this paper is that a novel idea of clustering, DSHTBMC, is proposed. Somehow like Shell-Sorting, which is a multi-stage sorting algorithm, DSHTBMC decomposes the task of clustering into multiple stages. Each stage is an easier clustering problem, and its solution can be used to induce solution of the next stage. In this way the initial clustering problem is eased. In contrast with traditional clustering algorithms through which the underlying data set remains changeless, homeomorphism transformation is employed in DSHTBMC. Two homeomorphism transformation strategies are introduced, and as an implementation of DSHTBMC, HD-DSHTBMC is proposed with Bisecting k-means as the subordinate algorithm. Experimental results show the effectiveness of DSHTBMC. Further works include designing diversified homeomorphism transformation strategies, diverse implementations of DSHTBMC, and employing various subordinate algorithms for different purposes. 


\section{References}

1. Pavel Berkhin: Survey of Clustering Data Mining Techniques. Technical report, Accrue Software (2002)

2. Qian Wei-Ning, Zhou Ao-Ying: Analyzing Popular Clustering Algorithms from Different Viewpoints. Journal of Software (2002)1382-1394

3. Hartigan J, Wong M.: A K-means Clustering Algorithm. Applied Statistics (1979)100-108

4. Raymond T.Ng, Jiawei Han: Efficient and Effective Clustering Methods for Spatial Data Mining. Proceeding of the 20th VLDB Conference Santiago, Chile (1994)144-155

5. Ordonez C, Omiecinski E. FREM: Fast and Robust EM Clustering for Large Data Sets. In ACM CIKM Conference (2002)590-599. http://citeseer.ist.psu .edu/536108.html

6. Karypis G, Han EH, Kumar V. CHAMELEON: A Hierarchical Clustering Algorithm Using Dynamic Modeling. COMPUTER (1999)68-75

7. Zhang T, Ramakrishna R, Livny M. BIRCH: A New Data Clustering Algorithm and its Applications. Journal of Data Mining and Knowledge Discovery (1997)141-182

8. Boley DL.: Principal Direction Divisive Partitioning. Technical Report TR-97-056, Dept. of Computer Science, University of Minnesota, Minneapolis, to appear in Data Mining and Knowledge Discovery (1997)

9. Boley DL.: Principal Direction Divisive Partitioning. Data Mining and Knowledge Discovery (1998)325-344

10. Sheikholeslami G, Chatterjee S, Zhang A. WaveCluster: A Multi-resolution Clustering Approach for Very Large Spatial Databases. In .Proceedings of the 24th Conference on VLDB, New York, NY (1998)428-439

11. Wang W, Yang J, Muntz R. STING: A Statistical Information Grid Approach to Spatial Data Mining. In Proceedings of the 23rd Conference on VLDB, Athens, Greece (1997) 186-195

12. Agrawal R, Gehrke J, Gunopulos D, Raghavan P.: Automatic Subspace Clustering of High Dimensional Data for Data Mining Applications. In Proc.1998 ACM-SIGMOD Int. Conf. Management of Data (SIGMOD'98), Seattle, WA June (1998)94-105

13. Ester M, Kriegel HP, Sander J, Xu X.: A density-based algorithm for discovering clusters in large spatial database. In Proc.1996 Int. Conf.Knowledge Discovery and Data Mining (KDD'96), Portland, OR, Aug (1996)226-231

14. Ankerst M, Breunig M, Kriegel HP: Sander J. OPTICS: Ordering points to identify the clustering structure. In Proc.1999 ACM-SIGMOD Int.Conf Management of Data (SIGMOD’99), Philadelphia, PA, June (1999) 49-60

15. Sergio M. Savaresi, Daniel L. Boley. On Performance of Bisecting k-means and PDDP. In Proceedings of the 1st SIAM ICDM, Chicago, IL (2001)1-14

16. Sergio M. Savaresi etc. Choosing the Cluster to Split in Bisecting Divisive Clustering Algorithms. CSE Report TR 00-055, University of Minnesota (2000)

17. Jinkun Lin. Basic of Topology. Beijing: Science Press (2003)19-22 